\title{
REFERENCES
}

1. Walter Rudin, Analyticity and the maximum modulus principle, Duke Math. J. vol. 20 (1953) pp. 449-458.

2. - Research Problem 19, Bull. Amer. Math. Soc. vol. 60 (1954) p. 399.

3. - Boundary values of continuous analytic functions, Proc. Amer. Math. Soc. vol. 7 (1956) pp. 808-811.

4. - Subalgebras of spaces of continuous functions, Proc. Amer. Math. Soc. vol. 7 (1956) pp. 825-830.

UNIVERSITY OF ROCHESTER

\section{ON A CLASS OF UNIVERSAL ORDERED SETS}

\section{ELLIOTT MENDELSON}

An ordered set $B$ is said to be $\boldsymbol{\aleph}_{\alpha}$-universal if and only if every ordered set of power $\boldsymbol{\aleph}_{\alpha}$ is similar to a subset of $B$. Let $U_{\omega_{\alpha}}$ be the lexicographically ordered set of all sequences of 0 's and 1's of type $\omega_{\alpha}$; and let $H_{\alpha}$ be the subset of $U_{\omega_{\alpha}}$ consisting of all sequences $\left\{x_{\xi}\right\}_{\xi<\omega_{\alpha}}$ for which there is some $\xi_{0}<\omega_{\alpha}$ such that $x_{\xi_{0}}=1$ and, for $\xi>\xi_{0}, x_{\xi}=0$.

$H_{0}$, being countable, dense, and without first or last element, is similar to the set of rationals in their natural order, and therefore, is $\boldsymbol{\aleph}_{0}$-universal. Sierpiński [2] has shown (as a direct consequence of his theorem that $H_{\alpha+1}$ is an $\eta_{\alpha+1^{-}}$-set) that, for any $\alpha, H_{\alpha+1}$ is $\boldsymbol{\aleph}_{\alpha+1^{-}}$ universal. Gillman [1] has given a demonstration that, for any limit ordinal $\alpha, H_{\alpha}$ is $\boldsymbol{\aleph}_{\alpha}$-universal. The purpose of this note is to give a very simple proof of these results, which does not depend on the ordinal $\alpha$.

TheOREM. $H_{\alpha}$ is $\boldsymbol{\aleph}_{\alpha}$-universal.

Proof. Let $A$ be an ordered set of power $\boldsymbol{\aleph}_{\alpha}$. Fix some well-ordering $\left\{a_{\beta}\right\}_{\beta<\omega_{\alpha}}$ of $A$. Let $\prec$ denote the order in $A$. Define a function $\phi$ from $A$ into $H_{\alpha}$ in the following way. Let $a_{\tau}$ be an element of $A$, and $\beta<\omega_{\alpha}$. Then the $\beta$ th component $\phi_{\beta}\left(a_{\tau}\right)$ of $\phi\left(a_{\tau}\right)$ is defined by:

$$
\phi_{\beta}\left(a_{\tau}\right)= \begin{cases}1 & \text { if } \beta \leqq \tau \text { and } a_{\beta} \leqq a_{\tau}, \\ 0 & \text { otherwise. }\end{cases}
$$

Now, let $a_{\tau}$ and $a_{\sigma}$ be any elements of $A$, with $a_{\tau}<a_{\sigma}$. Clearly, if $\beta \leqq \sigma, \phi_{\beta}\left(a_{\sigma}\right) \geqq \phi_{\beta}\left(a_{\tau}\right)$. But, $\phi_{\sigma}\left(a_{\sigma}\right)=1$ and $\phi_{\sigma}\left(a_{\tau}\right)=0$. Hence, $\phi\left(a_{\tau}\right)$ pre-

Received by the editors March 27, 1958. 
cedes $\phi\left(a_{\sigma}\right)$ in $H_{\alpha}$. Thus, $\phi$ is a one-one order-preserving mapping of $A$ into $H_{\alpha}$. Q.E.D.

Note that $H_{0}$ is $\boldsymbol{\aleph}_{0}$-universal and of power $\boldsymbol{\aleph}_{0}$. Since $\bar{H}_{\alpha+1}=2 \boldsymbol{\aleph}_{\alpha}$, $H_{\alpha+1}$ is of power $\boldsymbol{\aleph}_{\alpha+1}$ if and only if $2 \boldsymbol{\aleph}_{\alpha}=\boldsymbol{\aleph}_{\alpha+1}$. Finally, for limit ordinals $\alpha, \bar{H}_{\alpha}=\sum_{\beta<\alpha} 2 \aleph_{\beta}$, and, therefore, $H_{\alpha}$ is of power $\boldsymbol{\aleph}_{\alpha}$ if and only if, for every $\beta<\alpha, 2 \aleph_{\beta} \leqq \boldsymbol{\aleph}_{\alpha}$ (and, hence, if $2 \boldsymbol{\aleph}_{\beta}=\boldsymbol{\aleph}_{\beta+1}$ for all $\beta<\alpha$ ). For $\alpha>0$, it seems to be an open problem to prove, without additional cardinality assumptions, the existence of an $\boldsymbol{\aleph}_{\alpha}$-universal ordered set of power $\boldsymbol{\aleph}_{\boldsymbol{\alpha}}$.

\section{BIBLIOGRAPHY}

1. L. Gillman, Some remarks on $\eta_{\alpha^{-}}$-sets, Fund. Math. vol. 43 (1956) pp. 77-82.

2. W. Sierpiński, Sur une proprieté des ensembles ordonnés, Fund. Math. vol. 36 (1949) pp. 56-67.

HARVARD UNIVERSITY 